\title{
The Relationship Between Neutrophil/Lymphocyte Ratio (NLR) and Mean Platelet Volume (MPV) with Microalbuminuria in Participants with Different Glucose Tolerances
}

\author{
Farkl Glukoz Toleranslarma Sahip Katılimcılarda No trofil/Lenfosit Oranı (NLO) ve Ortalama Trombosit Hacmi \\ $(M P V)$ ile Mikroalbu minu ri Arasındaki İ lişki \\ ${ }^{1}$ Hasan Sözel, ${ }^{2}$ Fatih Yılmaz
}

${ }^{1}$ Akdeniz University, Faculty of Medicine, Department of Internal Medicine, Antalya, Turkey

${ }^{2}$ Antalya Atatürk State Hospital, Clinic of Nephrology, Antalya, Turkey
Correspondence:

Hasan SÖZEL

Akdeniz University, Faculty of Medicine, Department of Internal Medicine, Antalya, Turkey e-mail: dr07hasan@hotmail.com

\section{Abstract}

Neutrophil/lymphocyte ratio (NLR) is a simple and inexpensive marker of subclinical inflammation for chronic diseases. Mean platelet volume (MPV) is a platelet function index. This study aimed to evaluate the relationship of NLR and MPV with microalbuminuria in participants with different glucose tolerances. 951 patients (male/female=302/649) were divided into five groups according to their oral glucose tolerance test (OGTT) results: group $1=$ normal glucose tolerance (NGT), group $2=$ impaired fasting glucose (IFG), group 3=isolated impaired glucose tolerance (IGT), group 4=both IFG and IGT, and group 5=type 2 diabetes mellitus (DM). Additionally, patients were divided into three groups according to their glycated hemoglobin (HbAlc) results: group $1=$ NGT, group $2=$ prediabetes, and group $3=$ type $2 \mathrm{DM}$. Outcomes were compared between groups. According to the OGTT, $\mathrm{HbAlc}$, and OGTT $+\mathrm{HbA1c}$ criteria, there was a significantly positive correlation of microalbuminuria with MPV and NLR in all DM patients $(\mathrm{p}<0.001)$. Additionally, according to the OGTT, HbAlc, and OGTT+HbAlc criteria, there was a significantly positive correlation between MPV and microalbuminuria in all NGT patients $(\mathrm{p}<0.001)$. According to the OGTT and OGTT $+\mathrm{HbA1c}$ criteria, there was no significant correlation between NLR and microalbuminuria in prediabetic patients $(\mathrm{p}>0.05)$; however, there was a significantly positive correlation between NLR and microalbuminuria in the group with HbAlc 5.7-6.49 (p<0.001). Except isolated IFG and IGT, there was a significantly positive correlation between MPV and microalbuminuria in all prediabetic patients according to the OGTT, HbAlc, and OGTT+HbAlc criteria $(\mathrm{p}<0.001)$. NLR and MPV levels may be reliable predictive markers for the detection of microalbuminuria in prediabetes and DM.

Keywords: Neutrophil/Lymphocyte Ratio, Mean Platelet Volume, Microalbuminuria, Prediabetes, Diabetes Mellitus.

Nötrofil/lenfosit oranı (NLO), kronik hastalıklar için subklinik inflamasyonun basit ve ucuz bir belirtecidir. Ortalama trombosit hacmi (MPV) bir trombosit fonksiyon indeksidir. Bu çalıșma, farklı glukoz toleranslarına sahip katılımcılarda NLO ve MPV’nin mikroalbüminüri ile ilişkisini değerlendirmeyi amaçladı. 951 hasta (erkek / kadın = 302/649) oral glukoz tolerans testi (OGTT) sonuçlarına göre beş gruba ayrıldı: grup $1=$ normal glikoz toleransı (NGT), grup 2 = bozulmuş açlık glikozu (BAG), grup $3=$ izole bozulmuş glukoz toleransı (BGT), grup $4=$ hem BAG hem de BGT ve grup $5=$ tip 2 diabetes mellitus (DM). Ek olarak, hastalar glikolize hemoglobin (HbAlc) sonuçlarına göre üç gruba ayrıldı: grup $1=$ NGT, grup 2 = prediyabet ve grup $3=$ tip 2 DM. Sonuçlar gruplar arasında karşılaştırıldı. OGTT, HbAlc ve OGTT + HbAlc kriterlerine göre tüm DM hastalarında mikroalbüminüri ile MPV ve NLO arasında anlamlı pozitif korelasyon vardı ( $<<0.001)$. Ayrıca OGTT, HbAlc ve OGTT + HbA1c kriterlerine göre tüm NGT hastalarında MPV ile mikroalbüminüri arasında anlamlı pozitif korelasyon vardı $(p<0.001)$. OGTT ve OGTT + HbAlc kriterlerine göre prediyabetik hastalarda NLO ile mikroalbüminüri arasında anlamlı bir iliski yoktu (p $>0.05)$; ancak HbAlc 5,7-6,49 olan grupta NLO ile mikroalbüminüri arasında anlamlı pozitif korelasyon vardı (p <0,001). İzole BAG ve BGT dıșında OGTT, HbA1c ve OGTT + HbA1c kriterlerine göre tüm prediyabetik hastalarda MPV ile mikroalbüminüri arasında anlamlı pozitif korelasyon vardı $(\mathrm{p}<0,001)$. NLO ve MPV seviyeleri prediyabet ve DM'de mikroalbüminüri tespiti için güvenilir prediktif belirteçler olabilir.

Anahtar Kelimeler: Nötrofil/Lenfosit Oranı, Ortalama Trombosit Hacmi, Mikroalbüminüri, Prediyabet, Diabetes Mellitus.

Received 23.06.2021 Accepted 26.07.2021 Online published 29.07.2021 


\section{Introduction}

Diabetes mellitus (DM) is a chronic, systemic disease characterized by severe microvascular and macrovascular complications. Its worldwide incidence is rapidly increasing; according to the International Diabetes Federation, there will be approximately 580 million people with T2DM by the year 2030(1). Prediabetes is a metabolic disease defined by impaired fasting glucose (IFG), impaired glucose tolerance (IGT), or both based on oral glucose tolerance test (OGTT) results (2). Prediabetes refers to the process of hyperglycemia from normal glucose tolerance (NGT) to DM. It is of clinical importance due to its number of cases, which is over 300 million worldwide, its association with microand macrovascular complications, and its progression to diabetes (3).

Microvascular complications include neuropathy, retinopathy, and nephropathy, while macrovascular complications include stroke, cardiovascular diseases, and peripheral vascular diseases (1). Diabetic nephropathy (DN) is observed in $25 \%-40 \%$ patients with DM $(4,5)$. Diagnosis and monitoring of DN progression is performed by detecting microalbuminuria in the urine (6). The relationship between chronic inflammation and the progression of DM and the development of its complications has been described $(7,8)$. Many inflammatory cytokines (interleukin-1, 6, 8, etc.) are also related to the pathogenesis of DN. However, the use of these inflammatory markers in daily practice is. During the inflammatory response, changes in the circulating leukocyte ratio are accompanied by neutrophilia and relative lymphopenia. According to recent studies; the neutrophil/lymphocyte ratio (NLR) is a marker of subclinical systemic inflammation for chronic diseases. NLR is also used as a prognosis predictor in cardiovascular diseases, chronic kidney disease (CKD), malignancies, and metabolic syndrome (9-12). NLR is also thought to be a marker of systemic inflammation at CKD and DN $(13,14)$. Low NLR can be used as a new marker of earlystage DN and lower hospitalization risk in diabetic patients receiving hemodialysis $(15,16)$.
Mean platelet volume (MPV) can be a guide for diseases associated with platelet production or destruction. MPV increases when thrombocyte production increases in the bone marrow. High MPV may be an indication of increased platelet destruction due to inflammation $(17,18)$. Patients with IGT and IFG have been reported to have increased MPV (19). MPV is higher in those having retinopathy or microalbuminuria in patients with DM (20).

It has been reported that microalbuminuria is a risk factor for vascular complications in patients with DM and even IGT $(21,22)$. However, few studies about the relationship of NLR and MPV with urinary albumin excretion (UAE) in individuals with different glucose tolerances. We planned this study to evaluate the association of NLR and MPV with microalbuminuria in participants with different glucose tolerances.

\section{Materials and Methods}

This was a retrospective, single-center, observational study. Between January 2015 and June 2020, OGTT was applied to 5853 patients in total in Akdeniz University Faculty of Medicine Internal Medicine outpatient clinic, and the microalbumin levels of $1120(19.1 \%)$ of them were also measured.Patients with type $1 \mathrm{DM}$, chronic liver disease, CKD (Estimated Glomerular Filtration Rate (eGFR) $<60 \mathrm{~mL} / \mathrm{min} / 1.73 \mathrm{~m}^{2}$, serum creatinine $>1.3 \mathrm{mg} / \mathrm{dL}$ and/or urine microalbuminuria $\geq 300 \mathrm{mg} / \mathrm{g} \mathrm{Cr}$ ), gestational diabetes, morbid obesity, acute and chronic ischemic heart disease, active infection, acute massive bleeding, intoxication, malignancy, nephrotic syndrome causing urinary protein excretion, hematuria, renal vascular disease, dehydration, platelets, neutrophils, patients with hematological diseases or drug use that may affect lymphocyte production, and patients whose data was not available were not included in the study. A total of 951 patients $($ male/female $=302 / 649)$ were included in the study, with 169 patients excluded due to exclusion criteria.

Based on the diagnostic criteria for DM specified by the World Health Organization, 
the patients were divided into five groups (NGT, IFG, IGT, both IFG and IGT, and DM) according to their OGTT results (2). Additionally, the patients were divided into NGT $(\mathrm{HbA} 1 \mathrm{c}<5.7 \%)$, prediabetes $(\mathrm{HbA} 1 \mathrm{c}$ $5.7 \%-6.49 \%$ ) and DM (Hbalc $\geq 6.5 \%$ ) groups according to their $\mathrm{HbAlc}$ results. Microalbuminuria and creatinine levels were evaluated from the first morning urine of the patients, and UAE of $30-300 \mathrm{mg} / \mathrm{g}$ Cr was evaluated as microalbuminuria.

OGTT procedure: OGTT and microalbumin measurement were performed at the same outpatient admission that remained open for 10 days. For OGTT, patients were tested in the biochemistry laboratory with $75 \mathrm{~g}$ glucose and $0.5 \mathrm{~L}$ water, without having any food or drink on the day of the test. Before the patients were given the glucose solution, blood sample were taken, which was recorded at hour 0 . Patients with glucose level $\geq 126$ $\mathrm{mg} / \mathrm{dL}$ were considered T2DM and the test was discontinued for them. Furthermore, $75 \mathrm{~g}$ glucose was administered to the patient within 5-10 minutes and glucose values were measured at the 1 st and 2 nd hour. The patient remained in a sitting position without any food intake during the test.

Laboratory data: All biochemical examinations were performed in the central laboratory of our hospital. In the venous blood serum samples, glucose was measured using the hexokinase enzymatic method and creatinine was measured using the Jaffe method. Additionally, albumin was measured using bromocresol green via a spectrophotometric method using Siemens Advia Chemistry XP (Siemens Healthcare Diagnostics, Forchheim, Germany). Glycated hemoglobin (Hbalc) was measured using high performance liquid chromatography (Bio-Rad Laboratories, Marnes-la-Coquette, France). Results were expressed as \% values. The eGFR was calculated using the formula CKDEPI 2009 (Chronic Kidney Disease Epidemiologic Collaboration) (23). Complete blood count was performed using Sysmex XN 1000 (Sysmex Corporation, Kobe, Japan). Low-density lipoprotein cholesterol (LDL), high-density lipoprotein cholesterol (HDL), and triglyceride levels were measured by enzymatic colorimetric method, C-reactive protein (CRP) by immunoturbidimetric method, and microalbuminuria by immunoturbidimetric method using Siemens Advia 2400 biochemistry autoanalyzer (Siemens Healthcare Diagnostics, Forchheim, Germany).

Ethics: Local ethics committee approval was obtained for the study (XXX Ethics Committee-08/07/2020/492). During the realization of this study, where informed consent was not obtained due to its retrospective nature; the principles of the Declaration of Helsinki and all applicable local regulations have been complied with.

Statistical analysis: IBM SPSS Statistics for Windows, Version 23.0 (IBM Corp., Armonk, NY) was used for statistical analysis. ShapiroWilk test was used to test normality. Median (min-max), mean $\pm \mathrm{SD}$, or $\mathrm{n}(\%)$ were used for the presentation of descriptive analyses. Mann-Whitney $U$ test, Student's $t$ test, and Pearson's chi-square test were used for the analysis of non-normally distributed numerical data, normally distributed numerical data, and categorical data, respectively. Kruskal-Wallis test was used for comparing nonparametric variables between groups. Bonferroni-Dunn test was used as a post hoc test for cases that had significant results, whereas one-way ANOVA with post hoc Tukey's honestly significant difference test was used for parametric variables. Spearman's correlation coefficient was applied for investigating the correlation between continuous variables. Multivariate analyses of independent predictors of microalbuminuria were performed using a binary logistic regression model. Odds ratio (OR) was reported with corresponding 95\% confidence intervals. A value of $p \leq 0.05$ was accepted as statistically significant. In tables; if there is a significant difference between any two groups, it was shown with different lower letters, if not, with the same lower letters.

\section{Results}

The mean age of the 951 patients was $50.77 \pm 13.23$ years; $31.8 \%$ (302 patients) were males and $68.2 \%$ (649 patients) were females. According to OGTT results, $29.3 \%(n=279)$ patients had NGT, 49\% $(\mathrm{n}=466)$ were 
prediabetic, and $21.6 \%(\mathrm{n}=206)$ were diabetic. were higher in the DM group $(\mathrm{p}<0.001)$ The mean age and percentage of male patients (Tables 1-2).

Table 1. Comparison of patients' characteristics according to OGTT groups

\begin{tabular}{|c|c|c|c|c|c|}
\hline Variables & Overall & NGT & Pre-diabetes & $\mathrm{DM}$ & $\begin{array}{c}\mathrm{p} \\
\text { values }\end{array}$ \\
\hline Number (\%) & $951(100)$ & $279(29.3)$ & $466(49)$ & $206(21.7)$ & - \\
\hline Age (years) & $51.12 \pm 13.04$ & $45.1 \pm 14.45^{\mathrm{a}}$ & $52.8 \pm 11.84^{\mathrm{b}}$ & $55.47 \pm 10.56^{\mathrm{c}}$ & $<0.001$ \\
\hline \multicolumn{6}{|l|}{ Gender } \\
\hline Male,n(\%) & $302(31.8)$ & $76(27.2)^{\mathrm{a}}$ & $132(28.3)^{\mathrm{a}}$ & $94(45.6)^{b}$ & $<0.001$ \\
\hline Female,n(\%) & $649(68.2)$ & $203(72.8)$ & $334(71.7)$ & $112(54.4)$ & \\
\hline Hemoglobin (g/dL) & $13.51 \pm 1.54$ & $13.14 \pm 1.69^{\mathrm{a}}$ & $13.52 \pm 1.41^{\mathrm{b}}$ & $13.99 \pm 1.47^{\mathrm{c}}$ & $<0.001$ \\
\hline Creatinine $(\mathrm{mg} / \mathrm{dL})$ & $0.75 \pm 0.17$ & $0.72 \pm 0.17^{\mathrm{a}}$ & $0.76 \pm 0.16^{\mathrm{b}}$ & $0.79 \pm 0.17^{\mathrm{c}}$ & $<0.001$ \\
\hline $\begin{array}{l}\text { eGFR } \\
\left(\mathrm{mL} / \mathrm{min} / 1.73 \mathrm{~m}^{2}\right)\end{array}$ & $\begin{array}{c}111.23 \pm 15.6 \\
5\end{array}$ & $118.31 \pm 17.14^{\mathrm{a}}$ & $109.53 \pm 14.05^{b}$ & $105.49 \pm 13.45^{\mathrm{c}}$ & $<0.001$ \\
\hline CRP (g/dL) & $\begin{array}{l}0.32(0- \\
30.39)\end{array}$ & $0.22(0-3.04)^{\mathrm{a}}$ & $0.32(0.01-30.39)^{\mathrm{a}, \mathrm{b}}$ & $0.37(0.04-4.79)^{\mathrm{b}}$ & 0.022 \\
\hline $\begin{array}{l}\text { Triglyserides } \\
(\mathbf{m g} / \mathbf{d L})\end{array}$ & $\begin{array}{l}135(33- \\
1265.54)\end{array}$ & $\begin{array}{c}116.89(33- \\
469)^{\mathrm{a}}\end{array}$ & $135(38-1265.54)^{b}$ & $160.73(45-1201)^{\mathrm{c}}$ & $<0.001$ \\
\hline LDL (mg/dL) & $134(31-270)$ & $\begin{array}{l}127(31- \\
248.91)^{\mathrm{a}}\end{array}$ & $133.7(53.8-270)^{\mathrm{a}, \mathrm{b}}$ & $141(48-229.1)^{b}$ & 0.033 \\
\hline HDL (mg/dL) & $\begin{array}{c}46.45(15.3- \\
121.1)\end{array}$ & $\begin{array}{c}47.33(23- \\
121.1)^{\mathrm{a}}\end{array}$ & $47.05(15.3-102.3)^{\mathrm{a}}$ & $44(30-68)^{b}$ & 0.002 \\
\hline $\operatorname{Albumin}(\mathrm{g} / \mathrm{dL})$ & $\begin{array}{l}4.46(2.63- \\
5.65)\end{array}$ & $4.42(3.64-4.9)$ & $4.49(3.71-5.65)$ & $4.45(2.63-5.14)$ & 0.113 \\
\hline $\begin{array}{l}\text { Urine microalbumin } \\
\text { (mg/day) }\end{array}$ & $\begin{array}{c}9.1(0- \\
165.01)\end{array}$ & $4.73(0-116.7)^{\mathrm{a}}$ & $10.15(0.1-165.01)^{b}$ & $13.59(0.1-130.5)^{\mathrm{c}}$ & $<0.001$ \\
\hline $\begin{array}{l}\text { Microalbuminuria, } \\
\text { n (\%) }\end{array}$ & $167(17.5)$ & $29(10.4)^{\mathrm{a}}$ & $89(19.1)^{b}$ & $49(23.8)^{\mathrm{c}}$ & $<0.001$ \\
\hline ANC (cells×109/L) & $\begin{array}{l}3.99(1.38- \\
15.16)\end{array}$ & $\begin{array}{l}3.86(1.48- \\
10.85)\end{array}$ & $4(1.59-15.16)$ & $4.1(1.38-7.28)$ & 0.114 \\
\hline ALC $\left(\right.$ cells $\left.\times 10^{9} / \mathrm{L}\right)$ & $\begin{array}{l}2.22(0.7- \\
5.31)\end{array}$ & $2.26(1.08-5)^{\mathrm{a}}$ & $2.24(0.86-5.31)^{\mathrm{a}}$ & $2.01(0.7-4.98)^{b}$ & 0.001 \\
\hline AMC $\left(\right.$ cells $\left.\times 10^{9} / \mathrm{L}\right)$ & $\begin{array}{c}0.42(0.17- \\
1.32)\end{array}$ & $\begin{array}{c}0.41(0.17- \\
1.32)\end{array}$ & $0.41(0.2-1.29)$ & $0.44(0.17-1.15)$ & 0.515 \\
\hline APC $\left(\right.$ cells $\left.\times 10^{9} / \mathrm{L}\right)$ & $260(50-799)$ & $\begin{array}{l}258(132- \\
434)^{\mathrm{a}, \mathrm{b}}\end{array}$ & $266(110-799)^{\mathrm{a}}$ & $248(50-560)^{b}$ & 0.028 \\
\hline NLR & $\begin{array}{l}1.79(0.54- \\
9.24)\end{array}$ & $\begin{array}{c}1.69(0.59- \\
6.03)^{\mathrm{a}}\end{array}$ & $1.76(0.55-9.24)^{\mathrm{a}}$ & $2.03(0.54-4.98)^{\mathrm{b}}$ & $<0.001$ \\
\hline MPV (fL) & $8.1(5.4-12.8)$ & $7.6(5.7-12)^{\mathrm{a}}$ & $8.1(5.4-12)^{b}$ & $9(6-12.8)^{c}$ & $<0.001$ \\
\hline \multicolumn{6}{|c|}{$\begin{array}{l}\text { OGTT, oral glucose tolerance test; NGT, normal glucose tolerance; DM; diabetes mellitus; eGFR, estimated glomerular } \\
\text { filtration rate; } C R P, C \text {-reactive protein; LDL, low-density lipoprotein; HDL, high-density lipoprotein; ANC, absolute } \\
\text { neutrophil count; ALC, absolute lymphocytecount; AMC, absolute monocyte count; APC, absolute platelet caunt; NLR, } \\
\text { Neutrophil/Lymphocyte Ratio; MPV, mean platelet volume }\end{array}$} \\
\hline
\end{tabular}

Table 2. Comparison of patients' characteristics according to OGTT sub groups

\begin{tabular}{lcccccr}
\hline \multicolumn{5}{c}{ Pre-diabetes } \\
\hline Variables & NGT & IFG & IGT & IFG+IGT & DM & $\begin{array}{c}\text { p } \\
\text { values }\end{array}$ \\
\hline Number (\%) & $279(29.3)$ & $211(22.2)$ & $77(8.1)$ & $178(18.7)$ & $206(21.7)$ & \\
\hline Age (years) & $45.1 \pm 14.45^{\mathrm{a}}$ & $50.82 \pm 11.88^{\mathrm{b}}$ & $51.16 \pm 13.7^{\mathrm{b}}$ & $55.86 \pm 10.25^{\mathrm{c}}$ & $55.47 \pm 10.56^{\mathrm{c}}$ & $<\mathbf{0 . 0 0 1}$ \\
\hline Gender & & & & & \\
\multicolumn{1}{c}{ Male,n(\%) } & $76(27.2)^{\mathrm{a}}$ & $57(27)^{\mathrm{a}}$ & $17(22.1)^{\mathrm{a}}$ & $58(32.6)^{\mathrm{a}, \mathrm{b}}$ & $94(45.6)^{\mathrm{b}}$ & $<\mathbf{0 . 0 0 1}$ \\
\multicolumn{1}{c}{ Female, $\mathbf{n}(\%)$} & $203(72.8)$ & $154(73)$ & $60(77.9)$ & $120(67.4)$ & $112(54.4)$ & \\
\hline Hemoglobin (g/dL) & $13.14 \pm 1.69^{\mathrm{a}}$ & $13.53 \pm 1.27^{\mathrm{a}, \mathrm{b}}$ & $13.19 \pm 1.63^{\mathrm{a}}$ & $13.66 \pm 1.42^{\mathrm{b}}$ & $13.99 \pm 1.47^{\mathrm{c}}$ & $<\mathbf{0 . 0 0 1}$ \\
\hline
\end{tabular}




\begin{tabular}{|c|c|c|c|c|c|c|}
\hline $\begin{array}{l}\text { Creatinine } \\
(\mathrm{mg} / \mathrm{dL})\end{array}$ & $0.72 \pm 0.17^{\mathrm{a}}$ & $0.75 \pm 0.14^{\mathrm{a}, \mathrm{b}}$ & $0.76 \pm 0.18^{b}$ & $0.76 \pm 0.18^{b}$ & $0.79 \pm 0.17^{b}$ & $<0.001$ \\
\hline $\begin{array}{l}\text { eGFR } \\
\left(\mathrm{mL} / \mathrm{min} / 1.73 \mathrm{~m}^{2}\right)\end{array}$ & $118.31 \pm 17.14^{\mathrm{a}}$ & $111.48 \pm 13.25^{b}$ & $110.74 \pm 16.2^{b, c}$ & $106.7 \pm 13.58^{\mathrm{c}}$ & $105.49 \pm 13.45^{\mathrm{c}}$ & $<0.001$ \\
\hline CRP (g/dL) & $0.22(0-3.04)$ & $\begin{array}{l}0.44(0.01- \\
30.39)\end{array}$ & $0.37(0.01-2.1)$ & $\begin{array}{l}0.24(0.02- \\
1.67)\end{array}$ & $\begin{array}{c}0.37(0.04- \\
4.79)\end{array}$ & 0.052 \\
\hline $\begin{array}{l}\text { Triglyserides } \\
(\mathrm{mg} / \mathrm{dL})\end{array}$ & $\begin{array}{l}116.89(33- \\
469)^{\mathrm{a}}\end{array}$ & $\begin{array}{l}123.53(38- \\
1265.54)^{\mathrm{a}, \mathrm{b}}\end{array}$ & $\begin{array}{c}141.25(47- \\
413)^{\mathrm{b}, \mathrm{c}}\end{array}$ & $\begin{array}{l}143.61(51- \\
743)^{\mathrm{c}}\end{array}$ & $\begin{array}{c}160.73(45- \\
1201)^{\mathrm{c}}\end{array}$ & $<0.001$ \\
\hline LDL (mg/dL) & $\begin{array}{l}127(31- \\
248.91)^{\mathrm{a}}\end{array}$ & $\begin{array}{l}138.7(53.8- \\
270)^{\mathrm{a}, \mathrm{b}}\end{array}$ & $\begin{array}{c}127.75(70.29- \\
259.9)^{\mathrm{a}, \mathrm{b}}\end{array}$ & $\begin{array}{c}136.32(55.7- \\
238)^{\mathrm{a}, \mathrm{b}}\end{array}$ & $\begin{array}{l}141(48- \\
229.1)^{\mathrm{b}}\end{array}$ & 0.048 \\
\hline HDL (mg/dL) & $\begin{array}{c}47.33(23- \\
121.1)^{\mathrm{a}}\end{array}$ & $48.2(18-83.3)^{\mathrm{a}}$ & $\begin{array}{c}44.9(21.9- \\
102.3)^{\mathrm{a}, \mathrm{b}}\end{array}$ & $\begin{array}{l}45.8(15.3- \\
72.4)^{\mathrm{a}, \mathrm{b}}\end{array}$ & $44(30-68)^{b}$ & 0.003 \\
\hline $\operatorname{Albumin}(\mathrm{g} / \mathrm{dL})$ & $4.42(3.64-4.9)$ & $\begin{array}{l}4.51(3.86- \\
5.65)\end{array}$ & $\begin{array}{l}4.47(3.71- \\
5.05)\end{array}$ & $\begin{array}{c}4.43(3.77- \\
5.16)\end{array}$ & $\begin{array}{l}4.45(2.63- \\
5.14)\end{array}$ & 0.195 \\
\hline $\begin{array}{l}\text { Urine } \\
\text { microalbumin } \\
\text { (mg/day) }\end{array}$ & $4.73(0-116.7)^{\mathrm{a}}$ & $\begin{array}{l}8.2(0.1- \\
165.01)^{b}\end{array}$ & $\begin{array}{c}10.43(0.1- \\
104.7)^{\mathrm{b}, \mathrm{c}}\end{array}$ & $\begin{array}{c}13(0.1- \\
123.2)^{\mathrm{c}, \mathrm{d}}\end{array}$ & $\begin{array}{c}13.59(0.1- \\
130.5)^{\mathrm{d}}\end{array}$ & $<0.001$ \\
\hline $\begin{array}{l}\text { Microalbuminuria, } \\
\text { n (\%) }\end{array}$ & $29(10.4)^{\mathrm{a}}$ & $37(17.5)^{\mathrm{a}}$ & $15(19.5)^{\mathrm{a}, \mathrm{b}}$ & $37(20.8)^{b}$ & $49(23.8)^{b}$ & $<0.001$ \\
\hline $\begin{array}{l}\text { ANC (cells } \times 10^{9} \\
\text { /L) }\end{array}$ & $\begin{array}{c}3.86(1.48- \\
10.85)^{\mathrm{a}}\end{array}$ & $\begin{array}{c}3.87(1.59- \\
10.67)^{\mathrm{a}}\end{array}$ & $\begin{array}{l}4.06(1.9- \\
11.15)^{\mathrm{b}}\end{array}$ & $\begin{array}{l}4.17(1.87- \\
15.16)^{\mathrm{a}, \mathrm{b}}\end{array}$ & $\begin{array}{l}4.1(1.38- \\
7.28)^{\mathrm{a}, \mathrm{b}}\end{array}$ & 0.023 \\
\hline $\begin{array}{l}\text { ALC (cells } \times 10^{9} \\
/ \mathrm{L})\end{array}$ & $2.26(1.08-5)^{\mathrm{a}}$ & $\begin{array}{c}2.34(1.07- \\
4.52)^{\mathrm{a}}\end{array}$ & $\begin{array}{c}2.19(1.09- \\
3.78)^{\mathrm{a}, \mathrm{b}}\end{array}$ & $\begin{array}{l}2.21(0.86- \\
5.31)^{\mathrm{a}, \mathrm{b}}\end{array}$ & $\begin{array}{c}2.01(0.7- \\
4.98)^{\mathrm{b}}\end{array}$ & 0.001 \\
\hline $\begin{array}{l}\text { AMC (cells } \times 10^{9} \\
/ L)\end{array}$ & $\begin{array}{c}0.41(0.17- \\
1.32)\end{array}$ & $0.4(0.2-0.9)$ & $\begin{array}{c}0.42(0.23- \\
1.29)\end{array}$ & $\begin{array}{l}0.43(0.2- \\
1.08)\end{array}$ & $\begin{array}{c}0.44(0.17- \\
1.15)\end{array}$ & 0.419 \\
\hline $\begin{array}{l}\text { APC }\left(\text { cells } \times 10^{9}\right. \\
/ \mathrm{L})\end{array}$ & $258(132-434)$ & 262(144-799) & $\begin{array}{c}272.5(134- \\
499)\end{array}$ & $\begin{array}{l}267(110- \\
493)\end{array}$ & $248(50-560)$ & 0.108 \\
\hline NLR & $\begin{array}{c}1.69(0.59- \\
6.03)^{\mathrm{a}}\end{array}$ & $\begin{array}{c}1.61(0.55- \\
7.26)^{\mathrm{a}}\end{array}$ & $\begin{array}{c}1.94(0.86- \\
3.81)^{\mathrm{b}, \mathrm{c}}\end{array}$ & $\begin{array}{l}1.8(0.67- \\
9.24)^{\mathrm{b}}\end{array}$ & $\begin{array}{c}2.03(0.54- \\
4.98)^{c}\end{array}$ & $<0.001$ \\
\hline MPV (fL) & $7.6(5 \cdot 7-12)^{\mathrm{a}, \mathrm{b}}$ & $\begin{array}{l}7.9(5.4- \\
11.6)^{\mathrm{b}, \mathrm{c}}\end{array}$ & $8.2(5.8-12)^{\mathrm{c}, \mathrm{d}}$ & $\begin{array}{l}8.4(5.9- \\
11.3)^{\mathrm{d}}\end{array}$ & $9(6-12.8)^{\mathrm{e}}$ & $<0.001$ \\
\hline
\end{tabular}

OGTT, oral glucose tolerance test; NGT, normal glucose tolerance; IFG, impaired fasting glucose; IGT, impaired glucose tolerance; DM, diabetes mellitus; eGFR, estimated glomerular filtration rate; $C R P, C$-reactive protein; $L D L$, low-density lipoprotein; HDL, high-density lipoprotein; ANC, absolute neutrophil count; ALC, absolute lymphocytecount; AMC, absolute monocyte count; APC, absolute platelet caunt; NLR, Neutrophil/Lymphocyte Ratio; $M P V$, mean platelet volume

Data are presented as mean $\pm S D$, median (min-max) or $n(\%)$. ANOVA, Kruskal-Wallis test, Pearson chi-square test. Different lowercase letters in a row indicate statistically significant difference between groups.

As we progressed from the NGT group to the prediabetic and diabetic groups, the levels of hemoglobin, creatinine, triglycerides, microalbuminuria, and MPV increased, whereas eGFR decreased $(\mathrm{p}<0.001)$. Additionally, the prevalence of microalbuminuria increased $(\mathrm{p}<0.001)$ (Table $1-2)$. CRP $(p=.022)$ and direct LDL $(p=0.033)$ levels of the DM group were higher than those of the NGT group. HDL $(p=0.002)$ and absolute lymphocyte count (ALC) $(\mathrm{p}=0.001)$ values of the DM group were lower than that of the other groups, whereas NLR $(p<0.001)$ of the DM group was higher than that of the other groups.

Using ROC analysis for microalbuminuria, the optimal cut-off point for MPV was $\geq 8.35$ (area under the curve $[\mathrm{AUC}]=0.723[95 \%$ CI:0.681-0.766, $\mathrm{p}<0.001]$, sensitivity $=70.1 \%$, specificity $=63.7 \%)$ (Figure 1$)$.

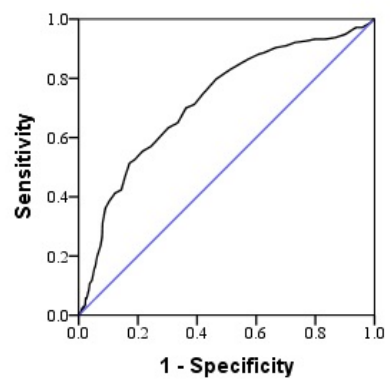

Figure 1. A ROC curve for MPV to predict microalbuminuria 
According to OGTT, 466 prediabetic patients were divided into three groups: IFG $(\mathrm{n}=211)$, IGT $(n=77)$, and IFG+IGT $(n=178)$. The mean ages of the IFG+IGT and DM groups were higher than those of the other three groups, and the mean ages of the IFG and IGT groups were higher than those of the NGT group $(p<0.001)$. The creatinine levels were higher in the IGT, IFG+IGT, and diabetic groups than in the NGT group $(\mathrm{p}<0.001)$. There was no difference between the eGFR values of the IGT, IFG+IGT, and DM groups, but the eGFR value of the IFG group was higher than that of the IFG+IGT and DM groups (Table 2).

Triglyceride levels of IFG+IGT and DM groups were higher than those of the NGT and IFG groups $(p<0.001)$. LDL levels of the DM group were higher than those of the NGT group ( $p=0.048)$. However, HDL levels of the NGT and IFG groups were higher than those of the DM group $(\mathrm{p}=0.003)$.

While the lowest median level of microalbuminuria was observed in the NGT group, the highest was in the DM group $(\mathrm{p}<0.001)$. The prevalence of microalbuminuria was higher in the IFG+IGT and DM groups compared to NGT and IFG groups $(\mathrm{p}<0.001)$. NLR was highest in the DM group and lowest in the NGT and IFG groups $(\mathrm{p}<.001)$. MPV increased as we progressed from NGT group to DM group $(p<0.001)$ (Table 2).

According to the HbAlc levels, the patients were divided into three groups: $<5.7(\mathrm{n}=336)$, 5.7-6.49 $(\mathrm{n}=531)$, and $\geq 6.5(\mathrm{n}=84)$. The mean age of the NGT group was the lowest $(p<0.001)$. The difference between the NLR levels of the HbAlc groups was not significant. The creatinine and triglyceride levels were higher and the HDL levels were lower in the $\mathrm{HbA} 1 \mathrm{c} \geq 6.5$ group compared to the other two groups (Table 3). The eGFR values of the $\mathrm{HbA} 1 \mathrm{c}<5.7$ group were higher than those of the other two groups $(p<0.001)$. As the HbAlc level increased, the level of microalbuminuria and the prevalence of MPV and microalbuminuria also increased $(p<0.001)$ (Table 3).

Table 3. Comparison of patients' characteristics according to HbAlc groups

\begin{tabular}{|c|c|c|c|c|}
\hline Variables & $<5.7$ & $5.7-6.49$ & $\geq 6.5$ & $\mathrm{p}$ values \\
\hline Number (\%) & $336(35.3)$ & $531(55.8)$ & $84(8.8)$ & \\
\hline Age (years) & $46.39 \pm 14.03^{\mathrm{a}}$ & $53.75 \pm 11.85^{b}$ & $53.4 \pm 10.67^{\mathrm{b}}$ & $<0.001$ \\
\hline \multicolumn{5}{|l|}{ Gender } \\
\hline Male,n(\%) & $101(30.1)^{\mathrm{a}}$ & $163(30.7)^{\mathrm{a}}$ & $38(45.2)^{b}$ & 0.020 \\
\hline Female,n(\%) & 235(69.9) & $368(69.3)$ & $46(54.8)$ & \\
\hline Hemoglobin (g/dL) & $13.55 \pm 1.65$ & $13.45 \pm 1.44$ & $13.75 \pm 1.72$ & 0.265 \\
\hline $\begin{array}{l}\text { Creatinine } \\
(\mathrm{mg} / \mathrm{dL})\end{array}$ & $0.74 \pm 0.16^{\mathrm{a}}$ & $0.76 \pm 0.17^{\mathrm{a}}$ & $0.81 \pm 0.18^{b}$ & 0.002 \\
\hline $\begin{array}{l}\text { eGFR } \\
\left(\mathrm{mL} / \mathrm{min} / 1.73 \mathrm{~m}^{2}\right)\end{array}$ & $116.01 \pm 16.17^{\mathrm{a}}$ & $108.93 \pm 14.56^{\mathrm{b}}$ & $106.63 \pm 15.66^{b}$ & $<0.001$ \\
\hline CRP (g/dL) & $0.29(0-30.39)$ & $0.32(0.01-4.79)$ & $0.58(0.07-4.2)$ & 0.171 \\
\hline $\begin{array}{l}\text { Triglyserides } \\
(\mathrm{mg} / \mathrm{dL})\end{array}$ & $123.83(33-654.98)^{\mathrm{a}}$ & $134.81(38-1265.54)^{\mathrm{a}}$ & $164(51-450)^{\mathrm{b}}$ & 0.003 \\
\hline LDL (mg/dL) & $132.7(32.6-270)$ & $133(31-247)$ & $143.8(58.66-206)$ & 0.427 \\
\hline HDL (mg/dL) & $45.2(21.9-121.1)^{\mathrm{a}}$ & $47.5(15.3-83.9)^{\mathrm{a}}$ & $41.1(30.3-64)^{b}$ & 0.002 \\
\hline $\operatorname{Albumin}(g / d L)$ & $4.46(3.71-4.9)$ & $4.46(3.44-5.65)$ & $4.44(2.63-5.05)$ & 0.981 \\
\hline $\begin{array}{l}\text { Urine } \\
\text { microalbumin } \\
\text { (mg/day) }\end{array}$ & $6.72(0.1-152.39)^{\mathrm{a}}$ & $10.5(0-165.01)^{b}$ & $19.15(0.1-128.12)^{\mathrm{c}}$ & $<0.001$ \\
\hline $\begin{array}{l}\text { Microalbuminuria, } \\
\text { n (\%) }\end{array}$ & $36(10.7)^{\mathrm{a}}$ & $103(19.4)^{b}$ & $28(33.3)^{\mathrm{c}}$ & $<0.001$ \\
\hline $\begin{array}{l}\text { ANC (cells } \times 10^{9} \\
/ L)\end{array}$ & $4.03(1.48-11.42)$ & $3.96(1.38-15.16)$ & $4.14(1.88-9.2)$ & 0.094 \\
\hline $\begin{array}{l}\text { ALC (cells } \times 10^{9} \\
\text { /L) }\end{array}$ & $2.22(0.91-4.2)$ & $2.21(0.7-5.31)$ & $2.29(1.11-4.98)$ & 0.990 \\
\hline
\end{tabular}




\begin{tabular}{lcccc}
\hline $\begin{array}{l}\text { AMC }\left(\text { cells } \times \mathbf{1 0}^{9}\right. \\
\text { /L) }\end{array}$ & $0.42(0.18-1.32)$ & $0.41(0.17-1.29)$ & $0.45(0.19-1.07)$ & 0.338 \\
APC $\left(\right.$ cells $\times \mathbf{1 0}^{9}$ & $254(50-799)$ & $264(89-493)$ & $256.5(108-560)$ & 0.533 \\
/L) & $1.81(0.59-7.26)$ & $1.75(0.54-9.24)$ & $1.95(0.85-4)$ & 0.351 \\
NLR & $7.6(5.7-12.8)^{\mathrm{a}}$ & $8.3(5.4-12.2)^{\mathrm{b}}$ & $9.3(6.1-11.7)^{\mathrm{c}}$ & $<\mathbf{0 . 0 0 1}$ \\
MPV (fL) & & &
\end{tabular}

HbAlc: glycated hemoglobin; eGFR, estimated glomerular filtration rate; CRP, C-reactiveprotein; LDL, low-density lipoprotein; HDL, high-density lipoprotein; ANC, absolute neutrophil count; ALC, absolute lymphocytecount; AMC, absolute monocyte count; APC, absolute platelet caunt; NLR, Neutrophil/Lymphocyte Ratio; MPV, mean platelet volume

Data are presented as mean $\pm S D$, median (min-max) or $n(\%)$. ANOVA, Kruskal-Wallis test, Pearson chi-square test. Different lowercase letters in a row indicate statistically significant difference between groups.

The patients were divided into groups as normal $(\mathrm{n}=146)$, prediabetes $(\mathrm{n}=569)$ and $\mathrm{DM}$ $(\mathrm{n}=236)$ according to OGTT results and/or HbA1c levels (Table 4). The mean age and ratio of male patients were the highest in the DM group $(p<0.001)$. Hemoglobin, CRP, triglyceride, and NLR levels of the DM group were higher, but ALC was lower, compared with the other groups (Table 4). The creatinine levels, microalbumin levels, MPV, and prevalence of microalbuminuria was higher, whereas the eGFR levels were lower $(p<0.001)$ in the DM group. The LDL levels of the DM group were higher than those of the NGT group. Additionally, HDL levels were higher in the prediabetes group than in the DM group (Table 4).

Table 4. Comparison of patients' characteristics according to OGTT-HbA1c combined groups

\begin{tabular}{|c|c|c|c|c|}
\hline Variables & NGT & Pre-diabetes & DM & $\mathbf{p}$ \\
\hline Number (\%) & $146(15.4)$ & $569(59.8)$ & $236(24.8)$ & \\
\hline Age (years) & $39.62 \pm 13.45^{\mathrm{a}}$ & $52.43 \pm 12.13^{b}$ & $55.08 \pm 10.84^{\mathrm{c}}$ & $<0.001$ \\
\hline \multicolumn{5}{|l|}{ Gender } \\
\hline Male,n(\%) & $38(26)^{\mathrm{a}}$ & $161(28.3)^{\mathrm{a}}$ & $103(43.6)^{b}$ & $<0.001$ \\
\hline Female,n(\%) & $108(74)$ & $408(71.7)$ & $133(56.4)$ & \\
\hline Hemoglobin (g/dL) & $13.36 \pm 1.79^{\mathrm{a}}$ & $13.38 \pm 1.44^{\mathrm{a}}$ & $13.91 \pm 1.56^{\mathrm{b}}$ & $<0.001$ \\
\hline $\begin{array}{l}\text { Creatinine } \\
(\mathrm{mg} / \mathrm{dL})\end{array}$ & $0.71 \pm 0.15^{\mathrm{a}}$ & $0.75 \pm 0.17^{b}$ & $0.79 \pm 0.17^{\mathrm{c}}$ & $<0.001$ \\
\hline $\begin{array}{l}\text { eGFR } \\
\left(\mathrm{mL} / \mathrm{min} / 1.73 \mathrm{~m}^{2}\right)\end{array}$ & $123.59 \pm 15.47^{\mathrm{a}}$ & $110.19 \pm 14.61^{b}$ & $106.1 \pm 14.23^{\mathrm{c}}$ & $<0.001$ \\
\hline CRP (g/dL) & $0.27(0-3.04)^{\mathrm{a}}$ & $0.28(0.01-30.39)^{\mathrm{a}}$ & $0.43(0.04-4.79)^{b}$ & 0.012 \\
\hline $\begin{array}{l}\text { Triglyserides } \\
(\mathrm{mg} / \mathrm{dL})\end{array}$ & $115(33-469)^{\mathrm{a}}$ & $130.98(38-1265.54)^{\mathrm{a}}$ & $160.68(45-1201)^{\mathrm{b}}$ & $<0.001$ \\
\hline LDL (mg/dL) & $125.36(32.6-248.91)^{\mathrm{a}}$ & $132.7(31-270)^{a, b}$ & $141.1(48-229.1)^{b}$ & 0.024 \\
\hline HDL (mg/dL) & $44.5(26.1-121.1)^{\mathrm{a}, \mathrm{b}}$ & $47.65(15.3-102.3)^{\mathrm{a}}$ & $43.7(30-68)^{b}$ & 0.001 \\
\hline $\operatorname{Albumin}(\mathrm{g} / \mathrm{dL})$ & $4.41(3.93-4.9)$ & $4.47(3.64-5.65)$ & $4.46(2.63-5.14)$ & 0.449 \\
\hline $\begin{array}{l}\text { Urine } \\
\text { microalbumin } \\
\text { (mg/day) }\end{array}$ & $3.3(0.1-63)^{\mathrm{a}}$ & $9(0-165.01)^{b}$ & $16.8(0.1-130.5)^{\mathrm{c}}$ & $<0.001$ \\
\hline $\begin{array}{l}\text { Microalbuminuria, } \\
\text { n (\%) }\end{array}$ & $12(8.2)^{\mathrm{a}}$ & $93(16.3)^{b}$ & $62(26.2)^{c}$ & $<0.001$ \\
\hline $\begin{array}{l}\text { ANC }\left(\text { cells } \times 10^{9}\right. \\
/ \mathrm{L})\end{array}$ & $4.13(1.48-10.85)$ & $3.96(1.56-15.16)$ & $3.99(1.38-9.2)$ & 0.176 \\
\hline $\begin{array}{l}\text { ALC }\left(\text { cells } \times 10^{9}\right. \\
/ L)\end{array}$ & $2.32(1.08-3.97)^{\mathrm{a}}$ & $2.23(0.86-5.31)^{\mathrm{a}}$ & $2.08(0.7-4.98)^{b}$ & 0.002 \\
\hline $\begin{array}{l}\text { AMC (cells } \times 10^{9} \\
/ \mathrm{L})\end{array}$ & $0.42(0.18-1.32)$ & $0.41(0.17-1.29)$ & $0.44(0.17-1.15)$ & 0.352 \\
\hline $\begin{array}{l}\text { APC (cells } \times 10^{9} \\
/ L)\end{array}$ & $257(132-433)$ & $263.5(110-799)$ & $251(50-560)$ & 0.136 \\
\hline NLR & $1.75(0.59-6.03)^{\mathrm{a}}$ & $1.72(0.55-9.24)^{\mathrm{a}}$ & $1.96(0.54-4.98)^{b}$ & 0.001 \\
\hline
\end{tabular}




\begin{tabular}{lllll}
\hline MPV (fL) & $7.1(5.7-10.6)^{\mathrm{a}}$ & $8(5.4-12)^{\mathrm{b}}$ & $9.1(6-12.8)^{\mathrm{c}}$ & $<\mathbf{0 . 0 0 1}$
\end{tabular}

HbAlc: glycated hemoglobin; OGTT, oral glucose tolerance test; NGT, normal glucose tolerance; DM; diabetes mellitus; eGFR, estimated glomerular filtration rate; CRP, C-reactive protein; LDL, low-density lipoprotein; HDL, high-density lipoprotein; $A N C$, absolute neutrophil count; $A L C$, absolute lymphocytecount; $A M C$, absolute monocyte count; APC, absolute platelet caunt; NLR, Neutrophil/Lymphocyte Ratio; MPV, mean platelet volume

Data are presented as mean $\pm S D$, median (min-max) or $n(\%)$. ANOVA, Kruskal-Wallis test, Pearson chi-square test. Different lowercase letters in a row indicate statistically significant difference between groups.

Microalbuminuria was detected in $17.5 \%$ $(\mathrm{n}=167)$ patients. Age, CRP, microalbumin, NLR, and MPV were higher, whereas ALC was lower in patients with microalbuminuria (Table 5). Other results have been presented in Table 5 .

Table 5. Comparison of patients' characteristics according to microalbuminuria

\begin{tabular}{|c|c|c|c|}
\hline Variables & Without & With & p values \\
\hline Number (\%) & $784(82.5)$ & $167(17.5)$ & \\
\hline Age (years) & $50.67 \pm 13.1$ & $52.81 \pm 12.7$ & 0.040 \\
\hline \multicolumn{4}{|l|}{ Gender } \\
\hline Male,n(\%) & $255(32.5)$ & $47(28.1)$ & 0.403 \\
\hline Female,n(\%) & $529(67.5)$ & $120(71.9)$ & \\
\hline Hemoglobin (g/dL) & $13.52 \pm 1.53$ & $13.48 \pm 1.55$ & 0.768 \\
\hline Creatinine (mg/dL) & $0.75 \pm 0.16$ & $0.76 \pm 0.18$ & 0.696 \\
\hline $\begin{array}{l}\text { eGFR } \\
\left(\mathrm{mL} / \mathrm{min} / 1.73 \mathrm{~m}^{2}\right)\end{array}$ & $111.62 \pm 15.62$ & $109.73 \pm 15.72$ & 0.130 \\
\hline CRP (g/dL) & $0.28(0-30.39)$ & $0.37(0.01-4.79)$ & 0.026 \\
\hline Triglyserides (mg/dL) & $131.75(33-779)$ & $147(45-1265.54)$ & 0.032 \\
\hline LDL (mg/dL) & $132.7(31-270)$ & $139.8(53.8-238)$ & 0.746 \\
\hline HDL (mg/dL) & $46.85(15.3-121.1)$ & $44.45(21.9-83.3)$ & 0.081 \\
\hline $\operatorname{Albumin}(\mathrm{g} / \mathrm{dL})$ & $4.45(3.64-5.14)$ & $4.48(2.63-5.65)$ & 0.721 \\
\hline $\begin{array}{l}\text { Urine microalbumin } \\
\text { (mg/day) }\end{array}$ & $6.5(0-19.87)$ & $35.85(20-165.01)$ & $<0.001$ \\
\hline ANC $\left(\right.$ cells $\left.\times 10^{9} / \mathrm{L}\right)$ & $3.96(1.38-15.16)$ & $4.14(1.94-11.15)$ & 0.064 \\
\hline ALC $\left(\right.$ cells $\left.\times 10^{9} / \mathrm{L}\right)$ & $2.25(0.88-5.31)$ & $2.1(0.7-4.24)$ & 0.001 \\
\hline AMC $\left(\right.$ cells $\left.\times 10^{9} / \mathrm{L}\right)$ & $0.41(0.17-1.32)$ & $0.43(0.18-1.29)$ & 0.502 \\
\hline APC $\left(\right.$ cells $\left.\times 10^{9} / \mathrm{L}\right)$ & $259(50-799)$ & $262(89-560)$ & 0.466 \\
\hline NLR & $1.74(0.54-9.24)$ & $2.05(0.7-5.25)$ & $<0.001$ \\
\hline MPV (fL) & $7.9(5.4-12.2)$ & $9.1(5.9-12.8)$ & $<0.001$ \\
\hline \multicolumn{4}{|c|}{$\begin{array}{l}\text { eGFR, estimated glomerular filtration rate; } C R P, C \text {-reactive protein; } L D L \text {, low-density lipoprotein; HDL, high } \\
\text { density lipoprotein; ANC, absolute neutrophil count; ALC, absolute lymphocytecount; AMC, absolute monocyt } \\
\text { count; APC, absolute platelet caunt; NLR, Neutrophil/Lymphocyte Ratio; } M P V \text {, mean platelet volume }\end{array}$} \\
\hline
\end{tabular}

In all models, MPV was identified as a predictive factor associated with microalbuminuria. In Model 1 (with 3 OGTT groups), MPV was positively associated with microalbuminuria (OR: 2.157; 95\% CI: 1.430-3.254; $\quad \mathrm{p}<0.001$ ) (Table 6). Additionally, patients with DM (OR: 5.822; 95\% CI: 1.226-27.641; $\mathrm{p}=0.027$ ) had higher risk of microalbuminuria compared to patients with NGT. In Model 2 (with 5 OGTT groups), increasing MPV (OR: 2.169; 95\% CI: 1.434 $3.279 ; \quad \mathrm{p}<0.001)$ was associated with microalbuminuria. Moreover, patients with DM were 5.76 times more likely to have microalbuminuria than patients with NGT (OR: 5.762; 95\% CI: 1.205-27.55; $\mathrm{p}=0.028$ ). In Model 3 (with HbA1c groups), increased MPV (OR: 2.303; 95\% CI: 1.535-3.456; $\mathrm{p}<0.001) \quad$ was associated with microalbuminuria. Patients with $\mathrm{HbA} 1 \mathrm{c}$ value 
between 5.7 and 6.5 (OR: 4.988; 95\% CI: 1.327-18.755; $\mathrm{p}=0.017$ ) or $\geq 6.5$ (OR: 23.444 ; 95\% CI: 3.634-151.244; $\mathrm{p}=0.001)$ had a higher risk of microalbuminuria compared to patients with NGT. In Model 4 (with OGTT or $\mathrm{HbA1c}$ groups), MPV was positively associated with microalbuminuria (OR: 2.098; 95\% CI: $1.446-3.042 ; \mathrm{p}<0.001)$. Patients with $\mathrm{DM}$ had a higher risk of microalbuminuria compared to patients with NGT (OR: 15.15; 95\% CI: 1.691-135.702; $\mathrm{p}=0.015$ ) (Table 6).

Table 6. Multivariate logistic regression analysis for microalbuminuria

\begin{tabular}{|c|c|c|c|c|c|c|c|c|}
\hline \multirow[b]{2}{*}{ Variables } & \multicolumn{2}{|c|}{$\begin{array}{l}\text { Model } 1 \text { with OGTT } \\
3 \text { groups }\end{array}$} & \multicolumn{2}{|c|}{$\begin{array}{l}\text { Model } 2 \text { with OGTT } \\
5 \text { groups }\end{array}$} & \multicolumn{2}{|c|}{$\begin{array}{c}\text { Model } 3 \text { HbA1c } \\
\text { groups }\end{array}$} & \multicolumn{2}{|c|}{$\begin{array}{c}\text { Model } 4 \text { with OGTT } \\
\text { or HbA1c groups }\end{array}$} \\
\hline & OR(95\%CI) & $\begin{array}{c}\text { p } \\
\text { values }\end{array}$ & OR(95\%CI) & $\begin{array}{c}\text { p } \\
\text { values }\end{array}$ & OR(95\%CI) & $\begin{array}{c}\mathbf{p} \\
\text { values }\end{array}$ & OR(95\%CI) & $\begin{array}{c}\mathbf{p} \\
\text { values }\end{array}$ \\
\hline Age (years) & $\begin{array}{c}1.015(0.976- \\
1.057)\end{array}$ & 0.456 & $\begin{array}{c}1.015(0.973- \\
1.058)\end{array}$ & 0.489 & $\begin{array}{c}1.014(0.974- \\
1.056)\end{array}$ & 0.493 & $\begin{array}{l}1.016(0.98- \\
1.052)\end{array}$ & 0.392 \\
\hline CRP & $\begin{array}{c}1.051(0.862- \\
1.28)\end{array}$ & 0.624 & $\begin{array}{c}1.046(0.858- \\
1.276)\end{array}$ & 0.655 & $\begin{array}{c}1.083(0.846- \\
1.387)\end{array}$ & 0.527 & $\begin{array}{c}1.053(0.884- \\
1.255)\end{array}$ & 0.560 \\
\hline Triglyserides & $\begin{array}{c}1.004(0.999- \\
1.009)\end{array}$ & 0.129 & $\begin{array}{c}1.004(0.999- \\
1.009)\end{array}$ & 0.135 & $\begin{array}{c}1.006(1- \\
1.012)\end{array}$ & 0.060 & $\begin{array}{c}1.001(0.998- \\
1.004)\end{array}$ & 0.359 \\
\hline HDL & $\begin{array}{c}1.018(0.98- \\
1.058)\end{array}$ & 0.367 & $\begin{array}{c}1.018(0.978- \\
1.059)\end{array}$ & 0.387 & $\begin{array}{c}1.022(0.98- \\
1.067)\end{array}$ & 0.309 & $\begin{array}{c}1.009(0.965- \\
1.025)\end{array}$ & 0.406 \\
\hline NLR & $\begin{array}{c}1.576(0.923- \\
2.691)\end{array}$ & 0.096 & $\begin{array}{c}1.619(0.926- \\
2.829)\end{array}$ & 0.091 & $\begin{array}{l}1.629(0.916- \\
2.895)\end{array}$ & 0.097 & $\begin{array}{c}1.154(0.733- \\
1.818)\end{array}$ & 0.536 \\
\hline MPV & $\begin{array}{c}2.157(1.43- \\
3.254)\end{array}$ & $<0.001$ & $\begin{array}{c}2.169(1.434- \\
3.279)\end{array}$ & $<0.001$ & $\begin{array}{l}2.303(1.535- \\
3.456)\end{array}$ & $<0.001$ & $\begin{array}{c}2.098(1.446- \\
3.042)\end{array}$ & $<0.001$ \\
\hline \multicolumn{9}{|l|}{$\begin{array}{l}\text { OGTT } 3 \\
\text { groups }\end{array}$} \\
\hline NGT & Reference & - & - & - & - & - & - & - \\
\hline $\begin{array}{l}\text { Pre- } \\
\text { diabetes }\end{array}$ & $\begin{array}{c}1.988(0.469- \\
8.423)\end{array}$ & 0.351 & - & - & - & - & - & - \\
\hline DM & $\begin{array}{c}5.822(1.226- \\
27.641)\end{array}$ & 0.027 & - & - & - & - & - & - \\
\hline \multicolumn{9}{|l|}{$\begin{array}{l}\text { OGTT } 5 \\
\text { groups }\end{array}$} \\
\hline NGT & - & - & Reference & - & - & - & - & - \\
\hline IFG & - & - & $\begin{array}{c}2.228(0.442- \\
11.225)\end{array}$ & 0.332 & - & - & - & - \\
\hline IGT & - & - & $\begin{array}{c}1.638(0.246- \\
10.913)\end{array}$ & 0.610 & - & - & - & - \\
\hline IFG+IGT & - & - & $\begin{array}{c}1.952(0.36- \\
10.581)\end{array}$ & 0.438 & - & - & - & - \\
\hline DM & - & - & $\begin{array}{c}5.762(1.205- \\
27.55)\end{array}$ & 0.028 & - & - & - & - \\
\hline \multicolumn{9}{|l|}{ HbA1c } \\
\hline$<5.7$ & - & - & - & - & Reference & - & - & - \\
\hline 5.7-6.49 & - & - & - & - & $\begin{array}{c}4.988(1.327- \\
18.755)\end{array}$ & 0.017 & - & - \\
\hline$\geq 6.5$ & - & - & - & - & $\begin{array}{c}23.444(3.634- \\
151.244)\end{array}$ & 0.001 & - & - \\
\hline \multicolumn{9}{|l|}{$\begin{array}{l}\text { OGTT or } \\
\text { HbA1c }\end{array}$} \\
\hline NGT & - & - & - & - & - & - & Reference & - \\
\hline $\begin{array}{l}\text { Pre- } \\
\text { diabetes }\end{array}$ & - & - & - & - & - & - & $\begin{array}{l}3.841(0.448- \\
32.949)\end{array}$ & 0.220 \\
\hline DM & - & - & - & - & - & - & $\begin{array}{c}15.15(1.691- \\
135.702)\end{array}$ & 0.015 \\
\hline $\begin{array}{l}\text { OGTT, oral glu } \\
\text { impaired fasting } \\
\text { Neutrophil/Lym }\end{array}$ & $\begin{array}{l}\text { tolerance tes } \\
\text { lucose; IGT, } \\
\text { cyte Ratio; MP }\end{array}$ & $\begin{array}{l}\text { HbAlc: } \\
\text { aired } \\
\text { mean p }\end{array}$ & $\begin{array}{l}\text { cated hemogl } \\
\text { ose toleranc } \\
\text { let volume }\end{array}$ & $N G 1$ & mal glucose & ince; & $\begin{array}{l}\text { M; diabetes mell } \\
\text { density lipoprote }\end{array}$ & $\begin{array}{l}\text { us; } I F G \\
n ; N L R\end{array}$ \\
\hline
\end{tabular}


According to the OGTT, HbAlc, and OGTT $+\mathrm{HbA1c}$ criteria, there was a significantly positive correlation of microalbuminuria with MPV and NLR in all DM patients $(p<0.001) \quad($ Table 7). Additionally, according to the OGTT, HbAlc, and OGTT $+\mathrm{HbAlc}$ criteria, there was a significantly positive correlation between MPV and microalbuminuria in all NGT patients $(\mathrm{p}<0.001)$. However, no significant correlation was found between NLR and microalbuminuria in any group (Table 7).
According to the OGTT and OGTT $+\mathrm{HbA1c}$ criteria, there was no significant correlation between NLR and microalbuminuria in prediabetic patients $(\mathrm{p}>0.05)$; however, there was a significantly positive correlation between NLR and microalbuminuria in the group with HbA1c 5.7-6.49 $(\mathrm{p}<0.001)$ (Table 7). Except isolated IFG and IGT, there was a significantly positive correlation between MPV and microalbuminuria in all prediabetic patients according to the OGTT, HbAlc, and OGTT + HbA1c criteria $(p<0.001)($ Table 7).

Table 7. Correlation of microalbumin with NLR and MPV in different glucose tolerance levels

\begin{tabular}{|c|c|c|c|c|}
\hline \multirow[b]{2}{*}{ Groups } & \multicolumn{2}{|c|}{ NLR } & \multicolumn{2}{|c|}{ MPV } \\
\hline & $\mathbf{r}$ & $\mathbf{p}$ & $\mathbf{r}$ & $\mathbf{p}$ \\
\hline \multicolumn{5}{|l|}{ OGTT 3 groups } \\
\hline NGT & 0.065 & 0.312 & 0.433 & $<0.001$ \\
\hline Pre-diabetes & 0.060 & 0.230 & 0.187 & $<0.001$ \\
\hline DM & 0.303 & $<0.001$ & 0.396 & $<0.001$ \\
\hline \multicolumn{5}{|l|}{ OGTT 5 groups } \\
\hline NGT & 0.065 & 0.312 & 0.433 & $<0.001$ \\
\hline IFG & 0.003 & 0.971 & 0.141 & 0.062 \\
\hline IGT & 0.129 & 0.288 & 0.213 & 0.079 \\
\hline IFG+IGT & 0.042 & 0.591 & 0.165 & 0.036 \\
\hline DM & 0.303 & $<0.001$ & 0.396 & $<0.001$ \\
\hline \multicolumn{5}{|l|}{ HbA1c } \\
\hline$<5.7$ & 0.024 & 0.700 & 0.406 & $<0.001$ \\
\hline $5.7-6.49$ & 0.203 & $<0.001$ & 0.238 & $<0.001$ \\
\hline$\geq 6.5$ & 0.326 & 0.005 & 0.441 & $<0.001$ \\
\hline \multicolumn{5}{|l|}{ OGTT and HbA1c } \\
\hline NGT & 0.052 & 0.574 & 0.366 & $<0.001$ \\
\hline Pre-diabetes & 0.081 & 0.070 & 0.153 & $<0.001$ \\
\hline DM & 0.288 & $<0.001$ & 0.423 & $<0.001$ \\
\hline \multicolumn{5}{|c|}{$\begin{array}{l}\text { OGTT, oral glucose tolerance test; NGT, normal glucose tolerance; IFG, impaired fasting glucose; IGT, impaired glucose } \\
\text { tolerance; DM; diabetes mellitus; HbAlc: glycated hemoglobin; CRP, C-reactive protein; HDL, high-density lipoprotein; NLR, } \\
\text { Neutrophil/Lymphocyte Ratio; MPV, mean platelet volume }\end{array}$} \\
\hline
\end{tabular}

\section{Discussion}

In our cohort study including 951 patients, we investigated the predictive value of hematological indices, such as NLR and $\mathrm{MPV}$, for predicting microalbuminuria in prediabetic patients. In our study, we found that NLR and MPV levels were significantly associated with decreased eGFR and increased UAE in prediabetes and DM patients.
Microalbuminuria is the earliest detectable sign of kidney damage in diabetic patients. In our study, as the glucose tolerance disorder progressed, the prevalence and level of microalbuminuria increased. In a study by Wang et al.(24) evaluating 2,394 individuals with different glucose tolerances, UAE was higher in the newly diagnosed T2DM, IFG + IGT, and isolated IGT groups compared to that of the isolated IFG and NGT group, with 
it being higher in the isolated IFG group than the NGT group.

In this study, MPV was significantly higher in patients with microalbuminuria than in patients without microalbuminuria, and there was a significantly positive correlation between MPV and microalbuminuria in all groups, except the IFG and IGT groups. Many studies have reported that prediabetic processes, such as IFG, IGT, and overt DM, are associated with increased MPV $(18,19,25)$. MPV has also been associated with vascular complications in patients with DM (26). Also, various studies report that MPV reflects many acute and cronic disease conditions, in addition to inflammatory burden or systemic inflammation (27). In a previous study in DM patients, MPV was found to be significantly higher in patients with poor diabetic control, and according to the results of the same study, MPV levels were also significantly positively correlated with UAE (28). In our study, when the MPV value was 8.35 , the sensitivity and specificity for microalbuminuria was $70.1 \%$ and $63.7 \%$, respectively.

MPV reflects a simple, fast, and easily attainable platelet size and prothrombotic potential. Platelets with increased MPV are hemostatically more active and show a stronger prothrombotic state with increased thromboxane A2 levels. However, MPV can be influenced by various factors (age, gender, blood pressure, and smoking). Increased MPV levels are a risk factor for atherosclerotic, cardiac, and cerebrovascular diseases. Large platelets are more active than normal sized platelets and secrete more prothrombotic factors. In this study, the difference between MPV levels in NGT, prediabetic, and diabetic groups was statistically significant. A significantly positive correlation was found between MPV and microalbuminuria, except for IFG and IGT. In multivariate logistic regression analysis, MPV was an independent risk factor for microalbuminuria in all groups. This may partially explain the increased risk of atherosclerosis and macrovascular complications during the prediabetic period.

NLR is an inexpensive indicator of systemic inflammation, which has been reported to be an inflammation marker in various studies. The systemic inflammatory response is often characterized by an increase in neutrophil count and a decrease in lymphocyte count. Leukocytes affect the initiation and progression of renal disease through noninfectious inflammatory mechanisms (such as free oxygen radicals and proteolysis in mesangial cells). As an indicator of inflammation, NLR shows a significant correlation with inflammation parameters. In our study, NLR increased significantly when we progressed from the NGT to the diabetic group. Additionally, CRP levels increased from the NGT to the DM group, which are an indicator of inflammation. NLR and microalbuminuria were positively correlated in the diabetic group and in those with $\mathrm{HbAlc}$ 5.7-6.4. In the multivariate logistic regression analysis, NLR was not considered as a significant risk factor for microalbuminuria $(p>0.05)$. With this result, our study differs from other studies in the literature. In the study of Shiny et al.(29), NLR was higher in patients with DM than in patients with IGT and NGT. Furthermore, NLR was positively correlated with fasting glucose and $\mathrm{HbAlc}$ levels, and it was stated that NLR could be used for detecting micro-and macrovascular complications (29). In another small-scale study, NLR was shown to has a positive predictive value for UAE in patients with DM (30). In another study involving geriatric patients with diabetes, NLR was indicated as a predictor for microvascular complications (28). In the study of Solak et al.(31), increased NLR was inversely related to reduced flowmediated dilatation and was reported to predict cardiovascular endpoints in patients with $\mathrm{CKD}$, regardless of traditional risk factors. Moreover, NLR has been identified as a predictor of CKD progression (13).

In conclusion, in all subgroups of prediabetic patients, MPV and NLR are higher than in the NGT group and lower than in the diabetic group. In the prediabetic groups, MPV and albuminuria had a significantly positive correlation. NLR and MPV levels may be reliable predictive markers for the detection of microalbuminuria in prediabetes and DM. Extensive prospective controlled studies with more subjects are required in the future to 
increase the use of NLR and MPV for predicting albuminuria.

Our study has some significant limitations. First, individuals with a history of obesity were excluded from the study, and anthropometric measurements, such as body mass index and abdominal circumference, could not be evaluated due to the lack of data on body composition. Second, although individuals taking medication or those with a history of hypertension were excluded from the study, the lack of data on blood pressure levels and antiplatelet drug use was another limitation. Third, we lacked data on liver function tests, family history of DM, smoking, and alcohol consumption; hence, evaluations related to these parameters could not be performed. Fourth, the cross-sectional nature of this study makes it difficult to interpret the relationship between NLR and MPV with

\section{REFERENCES}

1. Saeedi P, Petersohn I, Salpea P, et al IDF Diabetes Atlas Committee. Global and regional diabetes prevalence estimates for 2019 and projections for 2030 and 2045: Results from the International Diabetes Federation Diabetes Atlas, $9^{\text {th }}$ edition. Diabetes Res Clin Pract. 2019;157:107843.

2. Petersmann A, Müller-Wieland D, Müller UA, et al. Definition, classification and diagnosis of diabetes mellitus. Exp Clin Endocrinol Diabetes. 2019;127:1-7.

3. Cho NH, Shaw JE, Karuranga S, et al. IDF diabetes atlas: global estimates of diabetes prevalence for 2017 and projections for 2045. Diabetes Res Clin Pract. 2018;138:271-281.

4. Ritz E, Rychlik I, Locatelli F, et al. End-stage renal failure in type 2 diabetes: a medical catastrophe of worldwide dimensions. Am J Kidney Dis 1999;34:795808.

5. Juutilainen A, Lehto S, Rönnemaa T, et al. Similarity of the impact of type 1 and type 2 diabetes on cardiovascular mortality in middle-aged subjects. Diabetes Care. 2008;31:714-9.

6. Wang T, Wang Q, Wang Z, et al. Diagnostic value of the combined measurement of serum hcy, serum cys c, and urinary microalbumin in type 2 diabetes mellitus with early complicating diabetic nephropathy. ISRN Endocrinol. 2013;407452.

7. Kampoli A-M, Tousoulis D, Briasoulis A, et al. Potential pathogenic inflammatory mechanisms of endothelial dysfunction induced by type 2 diabetes mellitus. Curr Pharm Des. 2011;17:4147-4158.

8. Cebeci E, Cakan C, Gursu M, et al. The main determinants of serum resistin level in type 2 diabetic patients are renal function and inflammation not presence of microvascular complication, obesity and insulin resistance. Exp Clin Endocrinol Diabetes. 2019;127:189-194. microalbuminuria. Additionally, the change in microalbuminuria over time and the relationship between MPV and NLR were not determined. However, despite the study's retrospective nature, the study's major strength is the relatively large number of participants.

\section{Main Points}

In all subgroups of prediabetic patients, MPV and NLR are higher than in the NGT group and lower than in the diabetic group.

In the prediabetic groups, MPV and albuminuria had a significantly positive correlation.

NLR and MPV levels may be reliable predictive markers for the detection of microalbuminuria in prediabetes and DM.

9. Mei Z, Shi L, Wang B, et al. Prognostic role of pretreatment blood neutrophil-to-lymphocyte ratio in advanced cancer survivors: a systematic review and meta-analysis of 66 cohort studies. Cancer Treat Rev. 2017;58:1-13.

10. Woziwodzka K, Dziewierz A, Pawica $M$, et al. Neutrophil-to-lymphocyte ratio predicts long-term allcause mortality in patients with chronic kidney disease stage 5. Folia Med Cracov. 2019;59:55-70.

11. Liu CC, Ko HJ, Liu WS, et al. Neutrophil-tolymphocyte ratio as a predictive marker of metabolic syndrome. Medicine (Baltimore). 2019;98:e17537.

12. Afari ME and Bhat T. Neutrophil to lymphocyte ratio (NLR) and cardiovascular diseases: an update. Expert Rev Cardiovasc Ther. 2016;14:573-577.

13. Okyay GU, Inal $\mathrm{S}$, Oneç $\mathrm{K}$, et al. Neutrophil to lymphocyte ratio in evaluation of inflammation in patients with chronic kidney disease. Ren Fail. 2013;35:29-36.

14. Wu C-C, Sytwu H-K, and Lin Y-F. Cytokines in diabetic nephropathy. Adv Clin Chem. 2012;56:55-74.

15. Diaz-Martinez J, Campa A, Delgado-Enciso I, et al. The relationship of blood neutrophil-to-lymphocyte ratio with nutrition markers and health outcomes in hemodialysis patients. Int Urol Nephrol. 2019;51:12391247.

16. Khandare SA, Chittawar S, Nahar N, et al. Study of neutrophil-lymphocyte ratio as novel marker for diabetic nephropathy in type 2 diabetes. Indian $J$ Endocrinol Metab. 2017;21:387-392.

17. Bessman JD, Gilmer PR, and Gardner FH. Use of mean platelet volume improves detection of platelet disorders. Blood Cells. 1985;11:127-135.

18. Coban E, Bostan F, and Ozdogan M. The mean platelet volume in subjects with impaired fasting glucose. Platelets. 2006;17:67-69. 
19. Coban E, Kucuktag S, and Basyigit S. Platelet activation in subjects with impaired glucose tolerance. Platelets. 2007; 18:591-594.

20. Ünübol M, Ayhan M, and Güney E. The relationship between mean platelet volume with microalbuminuria and glycemic control in patients with type II diabetes mellitus. Platelets. 2012;23:475-480.

21. Dell'omo G, Penno G, Giorgi D, et al. Association between high-normal albuminuria and risk factors for cardiovascular and renal disease in essential hypertensive men. Am J Kidney Dis. 2002;40:1-8.

22. Garg JP and Bakris GL. Microalbuminuria: marker of vascular dysfunction, risk factor for cardiovascular disease. Vasc Med. 2002;7(1):35-43.

23. Levey AS, Stevens LA, Schmid $\mathrm{CH}$, et al. A new equation to estimate glomerular filtration rate. Ann Intern Med. 2009;150:604-612.

24. Wang XL, Lu JM, Pan CY, et al. A comparison of urinary albumin excretion rate and microalbuminuria in various glucose tolerance subjects. Diabet Med. 2005;22:332-335.

25. Zaccardi F, Rocca B, Pitocco D, et al. Platelet mean volume, distribution width, and count in type 2 diabetes, impaired fasting glucose, and metabolic syndrome: a meta-analysis. Diabetes Metab Res Rev. 2015;31:402410.

26. Patil P, Darshan A, Ao S, et al. Association of mean platelet volume with acute ischemic cerebrovascular accident among patients with type 2 diabetes mellitus: a hospital-based study. $J$ Assoc Physicians India. 2018;66:44-47.

27. Özer S, Yılmaz R, Sönmezgöz E, et al. Simple markers for subclinical inflammation in patients with familial mediterranean fever. Med Sci Monit. 2015;21:298-303.

28. Razak MKA, Akif AM, Nakeeb N, et al. The relationship between mean platelet volume and albuminuria in patients with type 2 diabetes mellitus. Diabetes Metab Syndr. 2019;13:2633-2639.

29. Shiny A, Bibin YS, Shanthirani CS, et al. Association of neutrophil-lymphocyte ratio with glucose intolerance: an indicator of systemic inflammation in patients with type 2 diabetes. Diabetes Technol Ther. 2014;16:524-530.

30. Assulyn T, Khamisy-Farah R, Nseir W, et al. Neutrophil-to-lymphocyte ratio and red blood cell distribution width as predictors of microalbuminuria in type 2 diabetes. J Clin Lab Anal. 2020;34:1-8.

31. Solak Y, Yilmaz MI, Sonmez A, et al. Neutrophil to lymphocyte ratio independently predicts cardiovascular events in patients with chronic kidney disease. Clin Exp Nephrol. 2013; 17:532-540.

๑Copyright 2022 by Osmangazi Tıp Dergisi - Available online at tip.ogu.edu.tr @Telif Hakkı 2022 ESOGÜ Tıp Fakültesi - Makale metnine dergipark.org.tr/otd web sayfasından ulaşılabilir. 